\title{
Continuous intraputamenal convection-enhanced delivery in adult rhesus macaques
}

\author{
Xiaotong Fan, MD, ${ }^{1,2}$ Brian D. Nelson, BS, ${ }^{4}$ Yi Ai, MD, ${ }^{2}$ David K. Stiles, PhD, ${ }^{4}$ Don M. Gash, PhD, ${ }^{2}$ \\ ${ }^{*}$ Peter A. Hardy, PhD, ${ }^{3,5}$ and Zhiming Zhang, MD² \\ 1Department of Neurosurgery, Xuan Wu Hospital, Capital Medical University, Beijing, China; ${ }^{2}$ Department of Anatomy \& \\ Neurobiology, College of Medicine, ${ }^{3}$ Magnetic Resonance Imaging and Spectroscopy Center, and ${ }^{5}$ Department of Radiology, \\ Chandler Medical Center, University of Kentucky, Lexington, Kentucky; and ${ }^{4}$ Medtronic Inc., Minneapolis, Minnesota
}

\begin{abstract}
OBJECT Assessing the safety and feasibility of chronic delivery of compounds to the brain using convection-enhanced delivery (CED) is important for the further development of this important therapeutic technology. The objective of this study was to follow and model the distribution of a compound delivered by CED into the putamen of rhesus monkeys.

METHODS The authors sequentially implanted catheters into 4 sites spanning the left and right putamen in each of 6 rhesus monkeys. The catheters were connected to implanted pumps, which were programmed to deliver a 5-mM solution of the MRI contrast agent Gd-DTPA at $0.1 \mu /$ minute for 7 days and $0.3 \mu /$ minute for an additional 7 days. The animals were followed for 28 days per implant cycle during which they were periodically examined with MRI.
\end{abstract}

RESULTS All animals survived the 4 surgeries with no deficits in behavior. Compared with acute infusion, the volume of distribution $\left(V_{d}\right)$ increased 2-fold with 7 days of chronic infusion. Increasing the flow rate 3-fold over the next week increased the $V_{d}$ an additional 3-fold. Following withdrawal of the compound, the half-life of Gd-DTPA in the brain was estimated as 3.1 days based on first-order pharmacokinetics. Histological assessment of the brain showed minimal tissue damage limited to the insertion site.

CONCLUSIONS These results demonstrate several important features in the development of a chronically implanted pump and catheter system: 1) the ability to place catheters accurately in a predetermined target; 2) the ability to deliver compounds in a chronic fashion to the putamen; and 3) the use of MRI and MR visible tracers to follow the evolution of the infusion volume over time.

http://thejns.org/doi/abs/10.3171/2015.1.JNS132345

KEY WORDS Parkinson disease; convection-enhanced delivery; rhesus macaques; magnetic resonance imaging

$\mathrm{T}$ HE blood-brain barrier (BBB) hinders the delivery of many promising drugs for the treatment of neurodegenerative disease. This is especially true for innovative new bio-therapeutics such as neurotrophic factors for treatment of Parkinson's disease or small interfering RNA (siRNA) for silencing the huntingtin gene (HTT). ${ }^{28,30}$ Given the tremendous potential of these therapies to treat degenerative CNS disorders, there is great interest in delivering them directly to the affected portion of the brain in order to slow or stop the disease progression. Such an approach would minimize side effects and would reduce the amount of drug needed to treat a condition.
Continuous convection-enhanced delivery (CED) into targeted brain areas has been tested in nonhuman primates (NHPs) for the treatment of various neurological diseases. . $^{10,12,17,22,26,30,31,38}$ Translational studies using CED have also gone into early clinical trials..$^{4,9,16,18,27,35}$ However, few CED studies have explored the challenges of designing hardware, along with the surgical procedures necessary for conducting chronic CED infusions into the brain. Additionally, developing techniques to measure the distribution of a therapeutic compound has become important. The challenge to do so is evident in the number of failed trials where the cause of the failure might have been an

ABBREVIATIONS BBB = blood-brain barrier; $C E D$ = convection-enhanced delivery; NHP = nonhuman primate; siRNA - small interfering RNA.

SUBMITTED October 24, 2013. ACCEPTED January 5, 2015.

INCLUDE WHEN CITING Published online May 1, 2015; DOI: 10.3171/2015.1.JNS132345.

DISCLOSURE Dr. Gash reports receiving support for this study from Medtronic. Dr. Stiles reports being a current employee of Pfizer and a former employee of Medtronic.

* Drs. Hardy and Zhang share senior authorship of this work. 
occluded delivery system, poor distribution of the therapeutic compound, or backflow around the catheter into the CSF spaces. ${ }^{23,24}$

Monitoring the distribution of therapeutic agents using MRI is valuable for confirming the operation of the infusion system and for accurately measuring the drug's efficacy. Emerging evidence suggests that functional improvement is often linked to drug distribution and drug effectiveness. ${ }^{1,7,8}$ To improve this technology we evaluated the feasibility of performing MRI monitoring of the MRI contrast agent Gd-DTPA during CED infusion in the brains of NHP. The infusion of this low-molecular-weight contrast agent was imaged during CED to assess its distribution in the brain as a function of infusion volume, infusion rate, and time. In the present study, normal adult rhesus macaques were tested with multiple continuous and chronic CED infusions of Gd-DTPA. MRI was used to monitor catheter patency, volume of distribution of the contrast agent, and kinetics of compound distribution and clearance.

\section{Methods}

\section{Experimental Animals and Design}

This study used 6 adult female rhesus monkeys (Macaca mulatta) (age range 8.5-15.5 years, weight 5.0-8.0 $\mathrm{kg}$ ) obtained from a commercial vendor (Covance). They were individually housed in temperature- and humiditycontrolled rooms, maintained on a 12-hour light/dark cycle. Experienced veterinarians worked closely with skilled laboratory technicians and animal caretakers to monitor the animals on a daily basis. Each animal's home cage activity and behavior were monitored daily to record the use of limbs, tactile/fine motor skills, visual tracking, lacrimation, startle response to noise, and salivation. The protocol for this study was approved by the Institutional Animal Care and Use Committee for the University of Kentucky. All procedures in this study were carried out in compliance with the Institute for Laboratory Animal Research's Guide for the Care and Use of Laboratory Animals. ${ }^{14}$

\section{Experimental Design}

The purpose of this study was to analyze targeted compound delivery into the primate putamen using a catheter designed to promote CED. The MRI contrast agent GdDTPA was used so that the infused compound could be tracked by noninvasive neuroimaging. ${ }^{13}$ The parameters evaluated were 1) neurological effects from continuous infusion into the putamen, 2) catheter placement, 3) catheter patency, 4) volume of distribution of 2 concentrations of test material, and 5) kinetics of compound distribution and clearance.

Each animal underwent 4 sequential catheter implantations into the putamen. The sequence of events is outlined in Fig. 1. Catheters were placed first in the right rostral putamen, followed by a left caudal putamen implantation, then third into the right caudal putamen, and finally into the left rostral putamen. Each infusion lasted a total of 14 days. The pump, anchor, and intraparenchymal catheter used are shown in Fig. 2. The anchor and catheter were removed immediately prior to each new implanta- tion; a single implanted pump was used for all 4 catheters. The multiple surgery strategy permitted repeated testing of catheter patency in the same animal. Anatomical MRI and postmortem tissue evaluation provided important information on how the brain handled multiple implantations to cover extensive target areas.

\section{Cage-Side Clinical Observation}

Throughout the study, animals were observed cageside at least once daily for abnormalities in motor function manifest in their gait, posture, or general appearance and to monitor water and food consumption and record any vomiting, seizures, or death. The animal's gaze, visual tracking, and pupil diameters were also observed to detect any neurological dysfunction. On observation days after surgery the incision sites were examined for any evidence of complications, such as bleeding, loose sutures, wound dehiscence. Observations were started 3 days prior to surgery and continued through the day of necropsy.

\section{Magnetic Resonance Imaging}

MRI scans were performed with a Siemens 3T Tim Trio using a receive-only coil custom built to conform to the animal's head to receive the nuclear magnetic resonance signal. For each imaging session the animal was anesthetized with ketamine and $1 \%-2 \%$ isoflurane, and its head was placed in an MRI-compatible stereotactic frame. During imaging sessions, the animal was kept warm with a combination of water-circulating heating pads and blankets. The animal's physiological state, including respiration, heart rate, peripheral capillary oxygen saturation $\left(\mathrm{SPO}_{2}\right)$ and exhaled $\mathrm{CO}_{2}$, were monitored continuously using a telemetry-operated physiological monitor (PRECESS, Invivo Corp.).

\section{Surgical Planning and Patency Determination}

For surgical planning, we used a 3D MPRAGE sequence with approximate $0.66-\mathrm{mm}$ isotropic resolution (TI $1100 \mathrm{msec}$, TR $2500 \mathrm{msec}$, TE $2.5 \mathrm{msec}$, flip angle $12^{\circ}$, image matrix size $174 \times 192 \times 115$, FOV $116 \times 128 \times$ $144 \mathrm{~mm}$ ) to visualize the putamen and to plan the coordinates of the target site for catheter placement.

\section{Catheter Replacement and Patency Determination}

Additional MRI scans were obtained approximately 30 minutes as well as 7 and 15 days after catheter implantation. These images were closely examined to measure the accuracy of the catheter placement, catheter patency, and tracer leak-back and to detect any tissue reaction to the placement of the catheter and infusion of the Gd-DTPA. As a result of the infusion of Gd-DTPA the implanted catheter patency was determined from the T1-weighted MR images by checking for high contrast signal surrounding the catheter tip. Structures surrounding the catheter tip were carefully examined by comparing their position and shape on postoperative MR images with those on images obtained before the surgery.

\section{Tracer Distribution}

To estimate the distribution of the tracer we acquired 


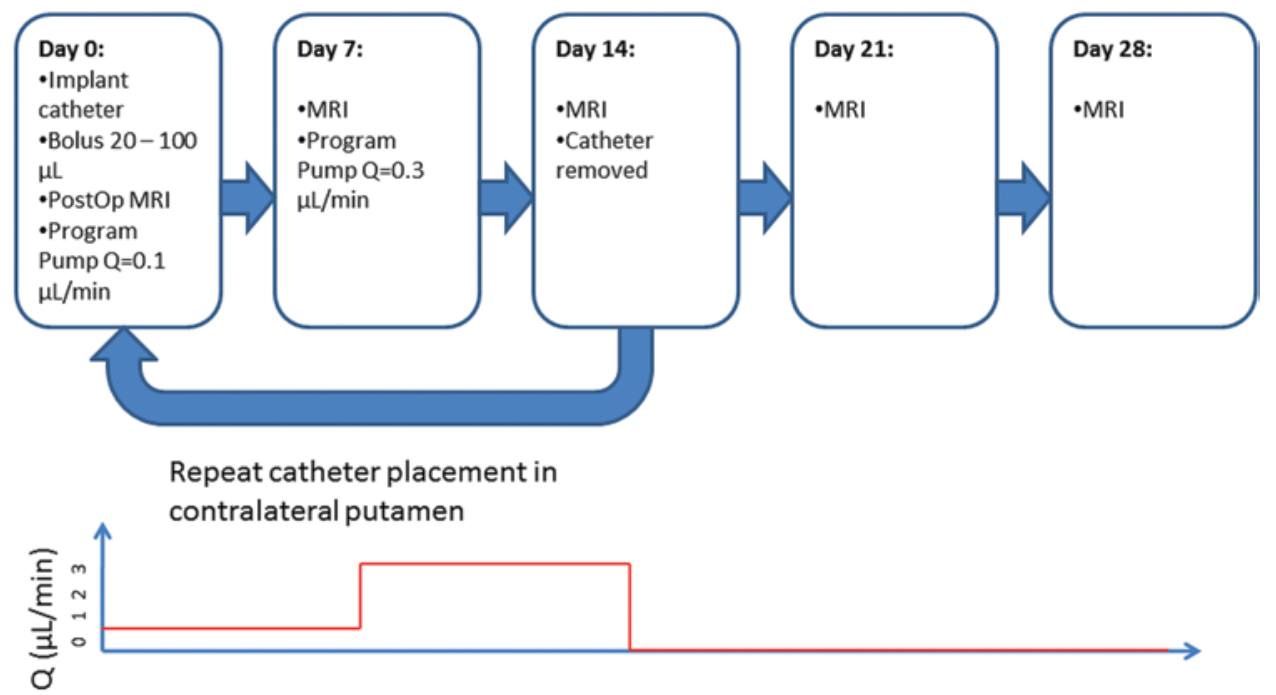

FIG. 1. Time line for placement of 1 of 4 catheters. Catheters implanted on Day 0 remained in place for 14 days. After Day 14 the catheter was removed, and in a repeat surgery a second (and ultimately a third and fourth) catheter was implanted. The site where a catheter had previously been implanted was examined on the MR images at Days 7 and 14 for the new catheter, which corresponded to Days 21 and 28 after the initial implantation. Figure is available in color online only.

a pair of T1-weighted spoiled gradient recalled acquisition (SPGR) images with the same TR and TE (20 and $3.8 \mathrm{msec}$, respectively) and spatial resolution but different flip angles. The flip angles $\left(\alpha=15^{\circ}\right.$ and $\left.\alpha=75^{\circ}\right)$ were selected according to the method of Deoni et al. to enable an accurate estimate of T1. ${ }^{6}$ From these images, the distribution and clearance of the Gd-DTPA were estimated (Fig. 3 and 4).

The volume of infusion $\left(V_{i}\right)$ was determined as the sum of the volumes infused during surgery and the product of the flow rate $(Q)$ and duration until the MR images were acquired. The volume of distribution $\left(V_{d}\right)$ was determined from a calculated relaxation rate $\mathrm{R} 1=1 / \mathrm{T} 1$ image derived by combining the signal from the pair of 3D gradient recalled echo images. ${ }^{34}$ Analysis of these calculated R1 images was conducted using a custom-developed computer program in which a total volume of distribution was derived by defining the area of enhanced $\mathrm{R} 1$ on each slice.

The volume of infusion over the 6 time points was modeled using first-order pharmacokinetics. The volume of distribution, $V_{d}(t)$, in the brain was the balance of material infused at a rate $Q$ and eliminated at a rate $k_{e l}$. The time variation $V_{d}(t)$ is given as

$$
\frac{d V_{d}(t)}{d t}=\beta Q-k_{e l} V_{d}(t)
$$

In Eq. $1, \beta$ is a constant that reflects the ratio of $V_{d}: V_{i}$. Equation 1 can be integrated from time $t=0$ when the volume is $V_{d}(0)$, i.e., immediately at the end of surgery, to time $\tau$ when the volume is $V_{d}(\tau)$. Then

$$
V_{d}(\tau)=\frac{\beta Q}{k_{e l}}\left[1-e^{-\tau k_{e l}}\right]+V_{d}(0) e^{-\tau k_{e l}}
$$

This model predicts an equilibrium volume at long times of $\beta Q / k_{e l}$.

\section{Surgical Procedures}

As described elsewhere, the chronic CED system used in the present study (see Fig. 2) is composed of a Medtronic SynchroMed II programmable pump (Model 8610-10), a catheter joining the pump to a cranial anchor (pump catheter), and a cranial catheter. ${ }^{11}$ Catheter patency was verified prior to insertion by observing flow from the exit holes. Infusing through the hollow stylet during implantation ensured positive flow out the exit holes, thereby preventing entrapment of blood or other tissue, and thus helped maintain patency. Under sterile field conditions, the cranial catheter was stereotactically placed on the dorsal edge of the putamen using a guide cannula and a 25-gauge hollow stylet, which was fully inserted into the distal catheter and connected to a syringe pump to infuse the Gd-DTPA solution at $1.0 \mu \mathrm{l} /$ minute. Then the catheter was slowly moved to the target $(3 \mathrm{~mm}$ above the ventral edge of the putamen). During the first of the 4 surgeries, the pump was subcutaneously implanted in the lateral abdominal region as previously described..$^{11,12}$ The pump was connected to the cranial anchor via the proximal catheter,

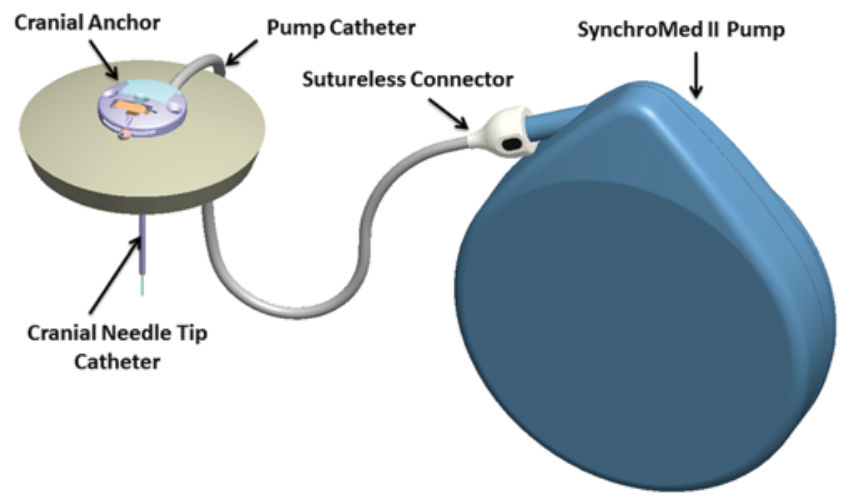

FIG. 2. SynchroMed II pump, cranial anchor, and catheter used in this study. Figure is available in color online only. 
which was primed with the Gd-DTPA solution prior to attachment to the pump. Once the pump and proximal catheter were implanted (for Sites 2-4, the pump was already implanted), the primed anchor was connected to the distal catheter and a single bolus of between 50-100 $\mu \mathrm{l}$ was delivered at $10 \mu \mathrm{l} /$ minute. At the end of this bolus the NHP was taken to the MRI area for its postoperative MRI scan.

\section{Histology}

At the end of the Trial 4 infusion, the brain of each NHP was removed and processed for routine histological evaluation. ${ }^{12}$ The entire striatum was cut into $40-\mu \mathrm{m}$-thick sections, and every 12th section was stained with hematoxylin and eosin and cresyl violet (Nissl) for gross and microscopic assessment of the tissue damage from the surgical procedures and drug infusion.

\section{Results \\ Cage-Side Clinical Observations}

All NHPs recovered from the surgeries without incident. Throughout the study, no obvious behavioral changes were found in any animal-including loss of consciousness of surroundings, inability to sit upright, impaired use of limbs or tactile/fine motor skills, impaired visual tracking, lacrimation, loss of startle response (to noise), abnormal salivation, or other impairments. All body weights remained uniform for the duration of the study, with an average deviation of $3.4 \%$.

\section{Catheter Replacement and Patency}

Catheters were targeted to 2 locations, one directly in front of the other, in the left and right putamen. All catheters but one were positioned inside the putamen as presurgically designed. The exception was a catheter that ended up outside of the boundaries of the putamen (approximately $1 \mathrm{~mm}$ in each of the 3 directions) due to accidental loosening of a screw on the head frame during surgery, resulting in movement of the head from the original stereotactic alignment. The mean radial deviation $( \pm$ $\mathrm{SD})$ of 23 out of 24 placements was $1.5 \pm 0.76 \mathrm{~mm}$ from the target. A typical target selected and catheter placement in the putamen with acute infusion is demonstrated in 3 dimensions in Fig. 5. No obvious structural changes, such as compression of the lateral ventricle, were seen when comparing MR images taken before and immediately after the surgical procedure.

\section{Patency}

Of the 24 catheters, the MR images demonstrated that 1 catheter was blocked permanently, which prevented Gd-DTPA from entering the putamen, and 1 catheter was temporarily blocked, with no Gd-DTPA entering the putamen until after the 7-day $0.1-\mu \mathrm{l} / \mathrm{min}$ infusion (Gd-DTPA was not seen in the Day 7 MRI but appeared in the Day 14MRI). Based on these 2 blockages, the surgical procedure was modified to directly connect the catheter system to an infusing SynchroMed II pump immediately after the needle tip catheter implant and to increase the volume of the bolus from $20 \mu \mathrm{l}(10 \mu \mathrm{l} / \mathrm{min}$ for 2 minutes) to $100 \mu \mathrm{l}$
(10 $\mu \mathrm{l} / \mathrm{min}$ for 10 minutes). These changes were employed for Sites 3 and 4 for a total of 12 infusions.

\section{Distribution of Gd-DTPA in the Brain}

In addition to checking catheter patency, we examined the immediate postoperative T1-weighted images for evidence of leak-back along the track of the catheter. We found evidence of minor leak-back, i.e., extending slightly beyond $50 \%$ of the catheter length in approximately $25 \%$ of the cases. A representative demonstration of the volume of distribution for the 5 time points sampled in the study is illustrated in Fig. 3. These sagittal MPRAGE images demonstrate the shape of the volume of distribution and the gradual expansion of the distribution with time. The last 2 images, acquired at Days 21 and 28, demonstrate the resolution of the infusion volume as the Gd-DTPA was cleared from the brain. By Day 28 there was little evidence of Gd-DTPA in the brain.

During the first round of surgeries the pumps were filled with a $10-\mathrm{mM}$ solution of Gd-DTPA. After viewing the images obtained immediately after surgery we decided to reduce the concentration of the solution to $5 \mathrm{mM}$ to reduce the intensity of the signal induced by the Gd-DTPA. The volumes derived from NHPs receiving the higher concentration of Gd-DTPA were excluded from further analysis, and all summary statistics were derived only from conditions using the 5-mM Gd-DTPA concentration. The values of $V_{i}, V_{d}$, and the ratio $V_{d}: V_{i}$ for the various time points are given in Table 1, which demonstrate how $V_{d}$ increased linearly with $V_{i}$. Immediately postoperatively, as indicated in Fig. 5, most of the infusate was contained within the putamen with some Gd-DTPA distributed outside the putamen (Fig. 5C). At this time point a dose-dependent increase of the $V_{d}: V_{i}$ ratio was found with a greater $V_{d}: V_{i}$ ratio $(7.4 \pm 3.2$ vs $2.1 \pm 1.4)$ seen in the sites with infusion volume of $147 \mu \mathrm{l}$ versus $62 \mu \mathrm{l}$. An incremental increase in $V_{d}$ was observed with an increase of the total $V_{i}$ and total infusion time. The volume of distribution increased more than 2-fold (1936 $\pm 660 \mu \mathrm{l}$ vs $818 \pm 233 \mu \mathrm{l})$ from the acute infusion immediately postsurgery to that after 7 days of continuous chronic infusion at a rate of $0.1 \mu \mathrm{l} /$ minute. The increase in $V_{d}$ over the 7 days from Day 7 to Day 14 compared with the increase between the immediate postoperative period and Day 7 was $2.9 \pm 1.3$. This is consistent with $V_{d}$ being proportional to the increase in infusion flow rate from $Q=0.1 \mu \mathrm{l} /$ minute to $0.3 \mu \mathrm{l} /$ minute. To better characterize this dependence, the data shown in Fig. 4 were fit to Eq. 2 using a least-squares approach. From this fitting, optimal values of the 3 fitting parameters $\beta, V_{d}(0)$, and $k_{e l}$ were derived as $3.25,837 \mu \mathrm{l}$, and $0.22 \mathrm{~L} / \mathrm{day}$. From these values, the data for the best-fit line was derived; it is shown in Fig. 4 as the smooth line passing through the data. From the estimate of $k_{e l}$ we derived a value of 3.1 days for the half-life of Gd-DTPA in the brain. Additionally, $\beta Q / k_{e l}=$ $6300 \mu \mathrm{l}$ is an estimate of the steady-state volume of distribution. From Fig. 4, this value is seen to be reasonable, as the average volume of distribution at Day 14 is $5250 \mu \mathrm{l}$ and the curve is clearly still rising.

\section{Histological Evaluation}

As shown in Fig. 6, the 4 infusion sites were clearly 

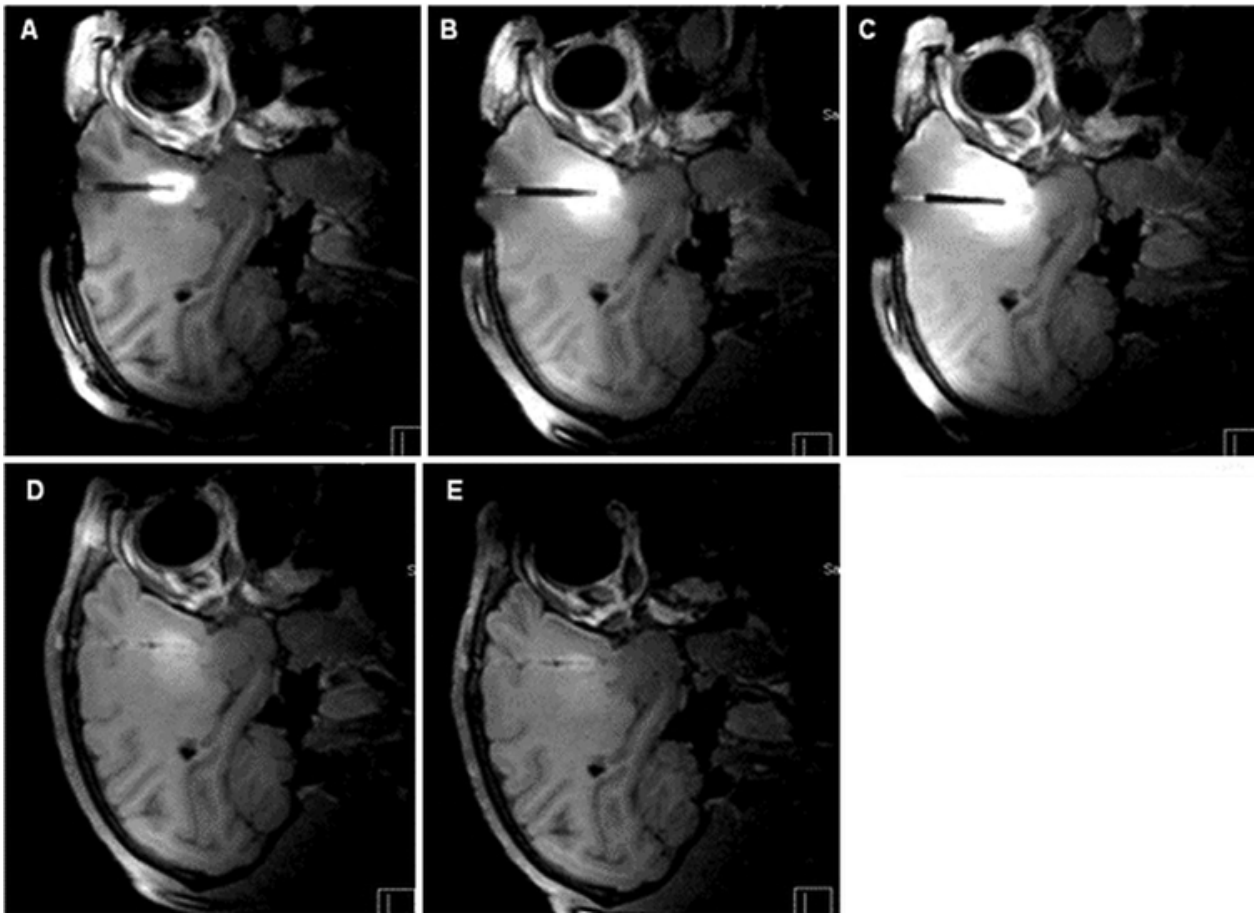

FIG. 3. A-C: Sagittal T1-weighted MR images of the brain of 1 NHP acquired 1 hour (A, Day 0), 7 days (B), and 14 days (C) after catheter implantation. D and E: Sagittal MR images acquired 7 days (D) and 14 days (E) after catheter withdrawal.

identified and verified to reside in the rostral and caudal putamen. With the exception of the disrupted tissue seen in Trial 4 (Fig. 6D), the tissue damage from the catheter placement and the infusion in the other 3 trials was limited (Fig. 6A-C). The excessive extracellular fluid is clearly visible in the infused putamen following the Trial 4 infusion in all the cases, but disappeared after the 2-week recovery following Trial 3 . In all the animals various amounts of mononuclear inflammatory cells were visible infiltrating the infusion site and the surrounding area. In some cases, small numbers of perivascular inflammatory infiltrates were seen in the infusion zone. Nissl staining

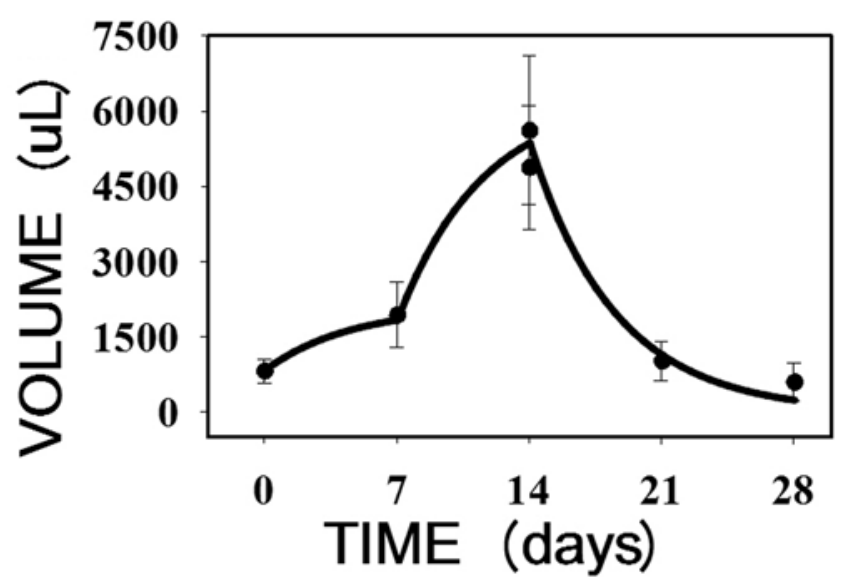

FIG. 4. Measured volume of distribution as a function of the time postimplantation. The data points represent the average volume calculated from measurements obtained in all animals that underwent infusion of 5-mM Gd-DTPA. The error bars represent standard deviations ( \pm 1 SD). The solid line represents the fit of Eq. 2 to the data. showed neuronal loss in the tissue along the catheter track and at the edge of the infusion site, but no detectable decline in neuronal population was observed in other areas.

\section{Discussion}

All animals tolerated the multiple surgeries for catheter implantation and continuous intraputamenal infusion at various flow rates. No obvious adverse effects on the NHPs, evidenced by cage-side observations, were noticed during the study. Overall outcomes support the contention that it is safe to use the catheter-pump system with chronic CED protocol for intraputamenal delivery of therapeutic agents. In addition, the results of the present study demonstrate that MRI is a valuable tool for visualizing the placement and patency of an implanted catheter and can supply important insights for better understanding of the infused testing agent's distribution in the basal ganglia.

TABLE 1. The volume of infusion $\left(V_{i}\right)$, volume of distribution $\left(V_{d}\right)$, and $V_{d} \cdot V_{i}$ ratio for all time points*

\begin{tabular}{crrc}
\hline Time & $V_{i}(\mu \mathrm{l})$ & \multicolumn{1}{c}{$V_{d}(\mu \mathrm{l})$} & $V_{d}: V_{i}$ \\
\hline Postop & 147 & $818 \pm 233$ & $5.56 \pm 1.6$ \\
\hline Day 7 & 1155 & $1936 \pm 660$ & $1.68 \pm 0.57$ \\
\hline Day 14- & 4179 & $5616 \pm 1480$ & $1.34 \pm 0.35$ \\
\hline Day 14+ & 4179 & $4877 \pm 1230$ & $1.17 \pm 0.29$ \\
\hline Day 21 & 4179 & $1018 \pm 400$ & $0.24 \pm 0.09$ \\
\hline Day 28 & 4179 & $598 \pm 380$ & $0.14 \pm 0.09$ \\
\hline
\end{tabular}

* The measurements for Day 14- were made from MR images acquired prior to the removal of the catheter. The measurements for Day 14+ were made after the removal of the catheter and using MR images acquired immediately after the insertion of a catheter into a new location to start the next trial. 

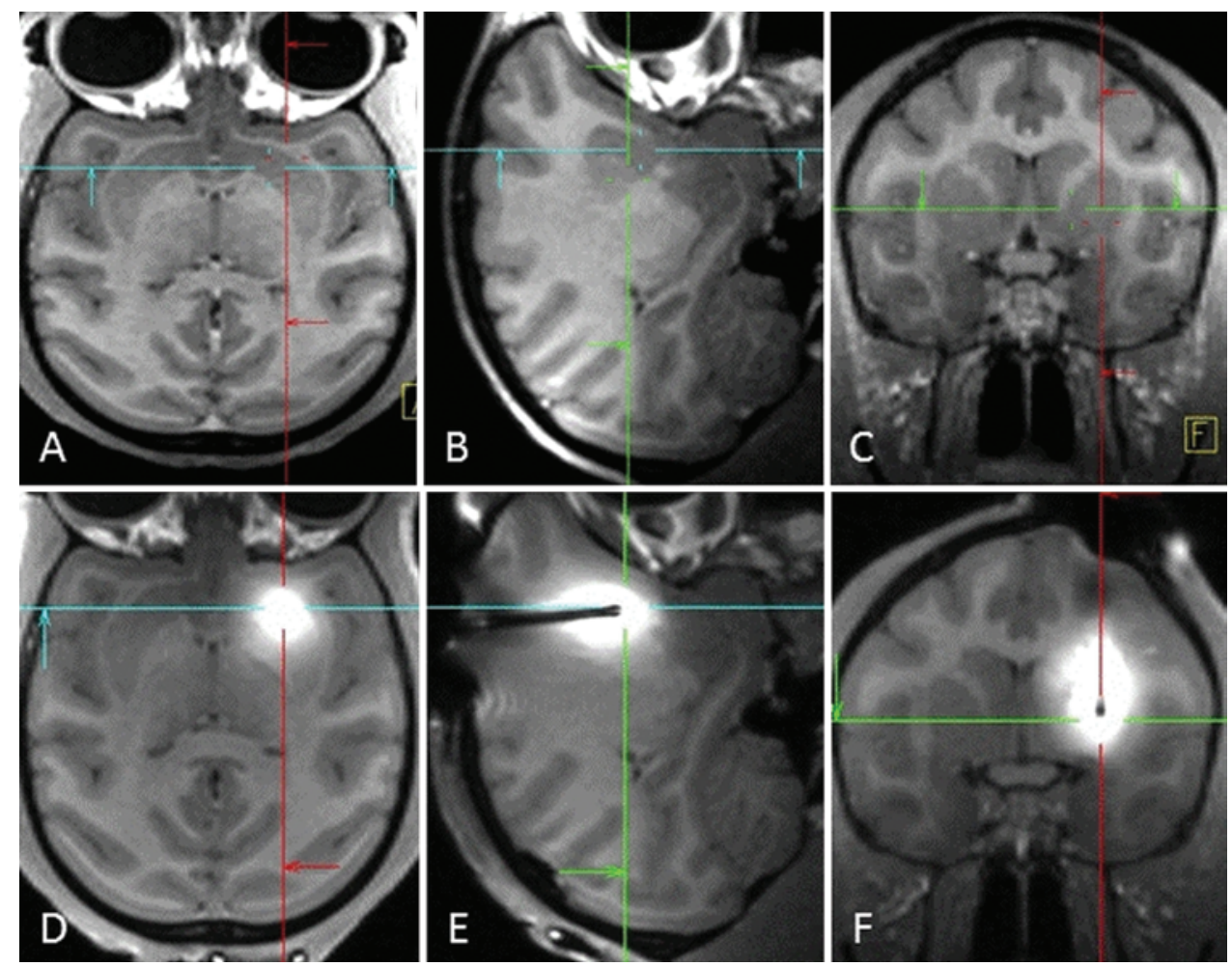

FIG. 5. A-C: Transverse (A), sagittal (B), and coronal (C) T1-weighted images obtained in 1 NHP showing the target location of the catheter in the center of the right putamen. D-F: Axial (D), sagittal (E), and coronal (F) T1-weighted images acquired immediately after catheter implantation showing the infusion of a test bolus of Gd-DTPA into the putamen. The distortion in the images at the top of the scalp arises from the presence of a metal anchor used to secure the implanted catheter and to join the catheter line coming from the pump to that inserted in the brain. The apparent dimensions of the catheter on these MR images are exaggerated as a result of partial volume effects and a small amount of susceptibility distortion. Figure is available in color online only.

Indeed, 2 blocked catheters were immediately identified, and one of them was confirmed to have reopened 7 days later. The study demonstrated one key advantage of utilizing a programmable pump - that the volume and flow rate can be adjusted based on demand. No blocked catheters were found during the phase of the study when the bolus volume delivered during implantation was increased from 20 to $100 \mu \mathrm{l}$.

This study used MRI to follow the distribution of the MR contrast agent, Gd-DTPA. The range of tracers used in CED studies is wide, including small-molecular-weight compounds such as Gd-DTPA, ${ }^{18,19,32}$ dendrimers, ${ }^{21}$ iron oxides, ${ }^{20,36}$ nanoparticles, ${ }^{33}$ and viruses ${ }^{5,32,33}$ with attached Gd moieties. The Gd-DTPA tracer is a small-molecularweight contrast agent with the widest FDA approval for use in CNS, body, and head and neck applications in both adults and children. Because our study was designed as a preclinical development we selected a tracer agent with the greatest likelihood of being approved for use as an indicator of patency.

The method of estimating the volume of distribution was made by using a calculated $\mathrm{T} 1$ image derived from a pair of gradient recalled echo images acquired at the same locations. This method enables an accurate estimate of the volume of drug delivered and is less dependent upon an arbitrary threshold in image intensity or selection of the concentration of the Gd-DTPA. ${ }^{2}$ T1-weighted images such as are produced by MPRAGE sequences can still be ana- lyzed but only after filtering to remove the spatial variation in signal intensity. The method is also likely to be more indicative of the distribution of an infused compound than is $\mathrm{T} 2$, which would demonstrate primarily the development of edema. ${ }^{15}$

The ratio of $V_{d}: V_{i}$ measured immediately after surgery was $5.56 \pm 1.6$, which then decreased to $1.68 \pm 0.57$ after 7 days of continuous infusion delivered an additional 1008 $\mu 1$. The ratio of $V_{d}: V_{i}$ declined further to $1.34 \pm 0.35$ after 14 days, with a total of $4179 \mu \mathrm{l}$ infused. The ratio of $V_{d}: V_{i}$ for the acute infusion found in this study is consistent with that found for acute infusion of a similar-sized MR contrast agent into the putamen of NHP. ${ }^{38}$ It is also consistent with measurements of a much larger molecule, ${ }^{123}$ I-labeled human serum albumin, which was infused into malignant glioma in patients. ${ }^{25}$ In that study the $V_{d}: V_{i}$ ratio after 48 hours was between 1.07 and 2.96, depending upon the isodose level used to define the distribution. Despite the consistency seen among these measurements, we expect that many factors-including the tissue in which the catheter is placed, the infusion rate, and the physical and chemical characteristics of the molecule infused (such as its molecular weight and its charge)-will affect distribution.

The results of the present study demonstrate that an incremental increase in the $V_{d}$ accompanied an increase of the total $V_{i}$ and the total infusion time during the first 7 days with continuous infusion at $0.1 \mu \mathrm{l} /$ minute. Then, over the next 7 days with the flow rate increased to $0.3 \mu \mathrm{l} /$ 


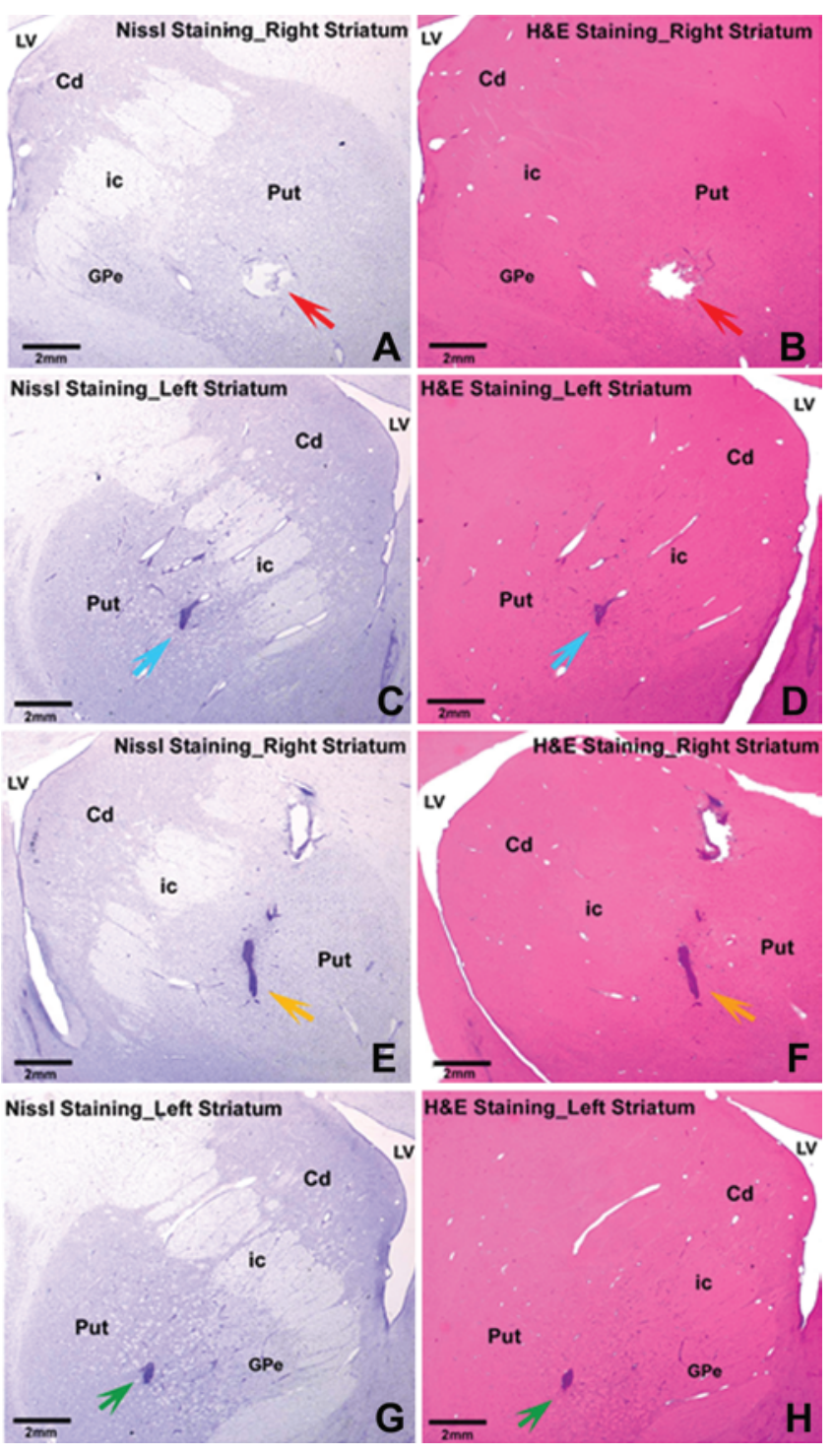

FIG. 6. Nissl- and H \& E-stained sections of the striatum at the site of catheter tip placement (arrows). The time points represented are 2 weeks (A and B), 4 weeks ( $C$ and D), 6 weeks ( $E$ and F), and 8 weeks ( $G$ and $\mathbf{H}$ ) after chronic infusion began. Acb = nucleus accumbens; $\mathrm{Cd}=$ caudate nucleus; $\mathrm{GPe}=$ globus pallidus externa; ic = internal capsule; LV $=$ lateral ventricle; Put = putamen. Figure is available in color online only.

minute, the average $V_{d}$ increased continuously, reaching a value 2.8 times higher than the value at Day 0 . Our results differ from those of a previous NHP study in which prolonged (up to 10 days) infusion of $\mathrm{Gd}+$ topotecan did not lead to a constant expansion of $V_{d}$, but rather maintained a constant volume. In the previous study, an open-tip catheter delivered a different material (Gd+topotecan) at a much higher flow rate of $0.486 \mu \mathrm{l} /$ minute into the brains of a different species (pigs). ${ }^{29}$ The results from this study suggest that different settings could lead to significant differences in clearance rates.

Theoretically, the bulk flow should follow the path of least resistance. Expansion outside the putamen will occur if the catheter tip is placed too close to white matter fiber tracks such as the corpus callosum. For example, in a GDNF study, backflow was only seen along the catheter tract passing the corpus callosum or very close to the internal capsule. ${ }^{2}$ Yin and colleagues also concluded that good distribution of infusates by CED depends very much on cannula design, precise cannula placement, and infusion rates. ${ }^{37}$ The most significant determinant of putamenal containment was the length of the cannula tip and the distance of the cannula step to the corpus callosum. ${ }^{37} \mathrm{In}$ the present study, we carefully selected the target area to be two-thirds down from the dorsal edge of the putamen; this ensured that the stepped portion of the needle tip was at least $2 \mathrm{~mm}$ into the putamen to avoid the infusate flowing back along the catheter.

Infusion rates could be another critical factor for successful induction of convection into the brain. The infused solution can leak out from the cannula track, resulting in a loss of infusion pressure, if the infusion rate is greater than a few microliters per minute. ${ }^{3}$ In the present study, we found that the tracer was contained inside the putamen at rates of 0.1 and $0.3 \mu \mathrm{l} / \mathrm{min}$, and the flow rate at $0.3 \mu \mathrm{l} / \mathrm{min}$ was used without any occurrence of backflow. Similar results were also seen in our previous studies of GDNF.,11,12 The histological findings indicated that the NHP brain can tolerate multiple surgical implants of the catheter as well as handle the flow rate and volume of the infusion through the putamenal catheter without observed behavioral change. Those results suggest that the flow rate could be used to achieve maximal distribution with minimal damage to the brain tissue.

Finally, MRI allowed the accurate determination of the volume of infusion at the 5 time points used in this study. By calculating the relaxation rate R1, we were able to derive a more accurate estimate of the volume of infusion than could be derived from image enhancement on MPRAGE images alone. From these estimates it is possible to characterize the kinetics of the infusion and clearance, which enables better prediction of the volume of distribution for flow rates and times outside those used in the study. Additionally, because the $V_{d}: V_{i}$ ratio declines with increasing $V_{i}$, the steady-state volume of distribution may become a more appropriate measure for chronic CED infusion with a long infusion period.

\section{Conclusions}

This work first demonstrated 1) the ability to accurately place a chronically implanted catheter in a predetermined target that is deep in the brain; 2) the use of MRI to visualize a MRI-visible tracer to follow the evolution of the infusion volume over time; and 3) the ability to safely deliver compounds in a chronic and continuous fashion in a large brain. The study supplied more insights into understanding fluid delivery and pharmacokinetic performance of a system used for chronic CED. The results suggest that the delivery system could be safe and effective in delivering therapeutic compounds in the large primate brain and could be translated into a clinical setting with some modifications for treatments of neurological disorders like Parkinson's disease and Huntington's disease.

\section{References}

1. Ai Y, Markesbery W, Zhang Z, Grondin R, Elseberry D, Ger- 
hardt GA, et al: Intraputamenal infusion of GDNF in aged rhesus monkeys: distribution and dopaminergic effects. J Comp Neurol 461:250-261, 2003

2. Asthagiri AR, Walbridge S, Heiss JD, Lonser RR: Effect of concentration on the accuracy of convective imaging distribution of a gadolinium-based surrogate tracer. J Neurosurg 115:467-473, 2011

3. Bobo RH, Laske DW, Akbasak A, Morrison PF, Dedrick RL, Oldfield EH: Convection-enhanced delivery of macromolecules in the brain. Proc Natl Acad Sci U S A 91:2076-2080, 1994

4. Carpentier A, Metellus P, Ursu R, Zohar S, Lafitte F, Barrié $\mathrm{M}$, et al: Intracerebral administration of $\mathrm{CpG}$ oligonucleotide for patients with recurrent glioblastoma: a phase II study. Neuro Oncol 12:401-408, 2010

5. Chen MY, Hoffer A, Morrison PF, Hamilton JF, Hughes J, Schlageter KS, et al: Surface properties, more than size, limiting convective distribution of virus-sized particles and viruses in the central nervous system. J Neurosurg 103:311319,2005

6. Deoni SC, Rutt BK, Peters TM: Rapid combined T1 and T2 mapping using gradient recalled acquisition in the steady state. Magn Reson Med 49:515-526, 2003

7. Gash DM, Zhang Z, Ai Y, Grondin R, Coffey R, Gerhardt GA: Trophic factor distribution predicts functional recovery in parkinsonian monkeys. Ann Neurol 58:224-233, 2005

8. Gash DM, Zhang Z, Cass WA, Ovadia A, Simmerman L, Martin D, et al: Morphological and functional effects of intranigrally administered GDNF in normal rhesus monkeys. J Comp Neurol 363:345-358, 1995

9. Gill SS, Patel NK, Hotton GR, O'Sullivan K, McCarter R, Bunnage M, et al: Direct brain infusion of glial cell linederived neurotrophic factor in Parkinson disease. Nat Med 9:589-595, 2003

10. Gimenez F, Krauze MT, Valles F, Hadaczek P, Bringas J, Sharma N, et al: Image-guided convection-enhanced delivery of GDNF protein into monkey putamen. Neuroimage 54 (Suppl 1):S189-S195, 2011

11. Grondin R, Zhang Z, Ai Y, Gash DM, Gerhardt GA: Intracranial delivery of proteins and peptides as a therapy for neurodegenerative diseases. Prog Drug Res 61:101-123, 2003

12. Grondin R, Zhang Z, Elsberry DD, Gerhardt GA, Gash DM: Chronic intracerebral delivery of trophic factors via a programmable pump as a treatment for parkinsonism. Methods Mol Med 62:257-267, 2001

13. Hardy PA, Keeley D, Schorn G, Forman E, Ai Y, Venugopalan R, et al: Convection enhanced delivery of different molecular weight tracers of gadolinium-tagged polylysine. $\mathbf{J}$ Neurosci Methods 219:169-175, 2013

14. Institute for Laboratory Animal Research: Guide for the Care and Use of Laboratory Animals, ed 8. Washington, DC: National Academies Press, 2011

15. Iyer RR, Butman JA, Walbridge S, Gai ND, Heiss JD, Lonser RR: Tracking accuracy of T2- and diffusion-weighted magnetic resonance imaging for infusate distribution by convection-enhanced delivery. J Neurosurg 115:474-480, 2011

16. Kunwar S, Chang S, Westphal M, Vogelbaum M, Sampson J, Barnett G, et al: Phase III randomized trial of CED of IL13-PE38QQR vs Gliadel wafers for recurrent glioblastoma. Neuro Oncol 12:871-881, 2010

17. Lang AE, Langston JW, Stoessl AJ, Brodsky M, Brooks DJ, Dhawan V, et al: GDNF in treatment of Parkinson's disease: response to editorial. Lancet Neurol 5:200-202, 2006

18. Lonser RR, Schiffman R, Robison RA, Butman JA, Quezado $\mathrm{Z}$, Walker ML, et al: Image-guided, direct convective delivery of glucocerebrosidase for neuronopathic Gaucher disease. Neurology 68:254-261, 2007

19. Lonser RR, Warren KE, Butman JA, Quezado Z, Robison RA, Walbridge S, et al: Real-time image-guided direct con- vective perfusion of intrinsic brainstem lesions. Technical note. J Neurosurg 107:190-197, 2007

20. Perlstein B, Ram Z, Daniels D, Ocherashvilli A, Roth Y, Margel S, et al: Convection-enhanced delivery of maghemite nanoparticles: increased efficacy and MRI monitoring. Neuro Oncol 10:153-161, 2008

21. Regino CA, Walbridge S, Bernardo M, Wong KJ, Johnson $\mathrm{D}$, Lonser R, et al: A dual CT-MR dendrimer contrast agent as a surrogate marker for convection-enhanced delivery of intracerebral macromolecular therapeutic agents. Contrast Media Mol Imaging 3:2-8, 2008

22. Saito R, Krauze MT, Bringas JR, Noble C, McKnight TR, Jackson P, et al: Gadolinium-loaded liposomes allow for realtime magnetic resonance imaging of convection-enhanced delivery in the primate brain. Exp Neurol 196:381-389, 2005

23. Salvatore MF, Ai Y, Fischer B, Zhang AM, Grondin RC, Zhang Z, et al: Point source concentration of GDNF may explain failure of phase II clinical trial. Exp Neurol 202:497505,2006

24. Sampson JH, Archer G, Pedain C, Wembacher-Schröder E, Westphal M, Kunwar S, et al: Poor drug distribution as a possible explanation for the results of the PRECISE trial. J Neurosurg 113:301-309, 2010

25. Sampson JH, Brady ML, Petry NA, Croteau D, Friedman $\mathrm{AH}$, Friedman HS, et al: Intracerebral infusate distribution by convection-enhanced delivery in humans with malignant gliomas: descriptive effects of target anatomy and catheter positioning. Neurosurgery 60 (2 Suppl 1):ONS89-ONS98

26. Sillay K, Schomberg D, Hinchman A, Kumbier L, Ross C, Kubota K, et al: Benchmarking the ERG valve tip and MRI Interventions Smart Flow neurocatheter convection-enhanced delivery system's performance in a gel model of the brain: employing infusion protocols proposed for gene therapy for Parkinson's disease. J Neural Eng 9:026009, 2012

27. Slevin JT, Gash DM, Smith CD, Gerhardt GA, Kryscio R, Chebrolu $\mathrm{H}$, et al: Unilateral intraputaminal glial cell linederived neurotrophic factor in patients with Parkinson disease: response to 1 year each of treatment and withdrawal. Neurosurg Focus 20(5):E1, 2006

28. Slevin JT, Gash DM, Smith CD, Gerhardt GA, Kryscio $\mathrm{R}$, Chebrolu H, et al: Unilateral intraputamenal glial cell line-derived neurotrophic factor in patients with Parkinson disease: response to 1 year of treatment and 1 year of withdrawal. J Neurosurg 106:614-620, 2007

29. Sonabend AM, Stuart RM, Yun J, Yanagihara T, Mohajed H, Dashnaw S, et al: Prolonged intracerebral convection-enhanced delivery of topotecan with a subcutaneously implantable infusion pump. Neuro Oncol 13:886-893, 2011

30. Stiles DK, Zhang Z, Ge P, Nelson B, Grondin R, Ai Y, et al: Widespread suppression of huntingtin with convection-enhanced delivery of siRNA. Exp Neurol 233:463-471, 2012

31. Sugiyama S, Saito R, Nakamura T, Yamashita Y, Yokosawa M, Sonoda Y, et al: Safety and feasibility of convectionenhanced delivery of nimustine hydrochloride co-infused with free gadolinium for real-time monitoring in the primate brain. Neurol Res 34:581-587, 2012

32. Szerlip NJ, Walbridge S, Yang L, Morrison PF, Degen JW, Jarrell ST, et al: Real-time imaging of convection-enhanced delivery of viruses and virus-sized particles. J Neurosurg 107:560-567, 2007

33. Valles F, Fiandaca MS, Eberling JL, Starr PA, Larson PS, Christine CW, et al: Qualitative imaging of adeno-associated virus serotype 2-human aromatic L-amino acid decarboxylase gene therapy in a phase I study for the treatment of Parkinson disease. Neurosurgery 67:1377-1385, 2010

34. Wang HZ, Riederer SJ, Lee JN: Optimizing the precision in T1 relaxation estimation using limited flip angles. Magn Reson Med 5:399-416, 1987 
35. White E, Bienemann A, Taylor H, Hopkins K, Cameron A, Gill S: A phase I trial of carboplatin administered by convection-enhanced delivery to patients with recurrent/progressive glioblastoma multiforme. Contemp Clin Trials 33:320-331, 2012

36. Writer MJ, Kyrtatos PG, Bienemann AS, Pugh JA, Lowe AS, Villegas-Llerena $\mathrm{C}$, et al: Lipid peptide nanocomplexes for gene delivery and magnetic resonance imaging in the brain. $\mathbf{J}$ Control Release 162:340-348, 2012

37. Yin D, Forsayeth J, Bankiewicz KS: Optimized cannula design and placement for convection-enhanced delivery in rat striatum. J Neurosci Methods 187:46-51, 2010

38. Yin D, Valles FE, Fiandaca MS, Bringas J, Gimenez F, Berger MS, et al: Optimal region of the putamen for image-guided convection-enhanced delivery of therapeutics in human and non-human primates. Neuroimage 54 (Suppl 1):S196-S203, 2011

\section{Author Contributions}

Conception and design: Zhang, Stiles, Gash. Acquisition of data: Zhang, Fan, Ai, Hardy. Analysis and interpretation of data: Zhang, Fan, Ai, Gash, Hardy. Drafting the article: Zhang, Hardy. Critically revising the article: Zhang, Stiles, Gash, Hardy. Reviewed submitted version of manuscript: Zhang, Gash, Hardy. Approved the final version of the manuscript on behalf of all authors: Zhang. Statistical analysis: Hardy. Administrative/technical/material support: Hardy. Study supervision: Zhang, Fan, Stiles, Gash. Provision of key components for surgery: Nelson.

\section{Correspondence}

Zhiming Zhang, Department of Anatomy \& Neurobiology, University of Kentucky College of Medicine, Medical Science Building, 800 Rose St., MN 208, Lexington, KY 40536-0298. email: zzhan01@uky.edu. 